MODELING, IDENTIFICATION AND CONTROL, 1996, VOL. 17, NO. 2, 75-86

doi:10.4173/mic.1996.21

\title{
Robust performance in dynamic positioning systems
}

\author{
ERLING JOHANNESSEN† and OLAV EGELAND†
}

Keywords: Dynamic positioning, surface vessels, robust performance, structured singular value, multivariable frequency analysis

\begin{abstract}
Robust performance of dynamic positioning of surface vessels is considered. The structured singular value, $\mu$, is used as a robustness measure. A tight uncertainty description of the wave disturbance model and performance weight matrices based on physical insight is presented. Two controllers are analysed: a decentralized PD controller with notch filters and an LQG based controller. Performance of the control systems is shown to be sensitive with respect to changes in the dominating wave frequency, $\omega_{0}$.
\end{abstract}

\section{Introduction and problem statement}

The purpose of a dynamic positioning system for surface vessels is to achieve a desired position and orientation of the vessel in the presence of disturbances from waves, wind and water currents (Morgan 1978, Fay 1990).

One of the main problems encountered in the design of dynamic positioning systems is to obtain high positioning accuracy without excessive thruster modulation due to wave induced motion.

Early implementations of dynamic positioning systems used a PD controller for each of the three degrees of freedom: surge, sway and yaw. Notch filters were used to reduce thruster modulation due to first order wave motion. Later, applications using state estimation and optimal control have been reported (Balchen et al. 1976, 1980, Sælid et al. 1983, Grimble and Patton 1980, Grimble et al. 1980).

Environment disturbances change over time. In particular, the dominating wave frequency may change as an offshore operation requiring dynamic positioning is in progress. Balchen et al. (1980) propose using a gradient adaptation algorithm to update the wave frequency estimate in the vessel model. Earlier work (Balchen et al. 1976) proposed using an augmented Kalman filter.

In the present paper the effect of changing dominating wave disturbance on control system performance is addressed. It is assumed that the wave frequency may vary in a band around a nominal value. It is shown how a tight uncertainty description in the wave disturbance model can be constructed. Both a traditional decentralized solution and an LQG based controller are considered.

Performance of multivariable linear control systems may be analysed in the frequency domain (Doyle and Stein 1981). With the introduction of the structured singular value ( $\mu$, SSV) (Doyle 1982), analysis of systems with a certain block diagonal structure is possible. Summaries of this theory may be found in Maciejowski (1989) and Skogestad (1994).

Received 20 August 1995.

† Department of Engineering Cybernetics. The Norwegian Institute of Technology, N-7034 Trondheim, Norway. (E-mail: Erling.Johannessen@itk.unit.no, Olav.Egeland@itk.unit.no)

Presented at the 3rd IFAC Workshop on Control Applications in Marine Systems, Trondheim, Norway, May 1995 (CAMS '95). 
The paper is organized as follows: first the vessel model is presented and an uncertainty description in the wave disturbance model is given. Then robust performance in the frequency domain is defined and two different control designs are analysed with respect to performance robustness.

\section{Vessel model}

The vessel model from Fossen (1994) is considered, and the following simplifications are made:

(S1) Water currents will not be considered.

(S2) Effects due to wind will not be considered.

(S3) Thruster dynamics are neglected.

(S4) Thrust allocation will be assumed perfect.

These simplifications are made only to be able to focus on uncertainty (variations) in the wave frequency.

Three degrees of freedom are of importance in dynamic positioning:

- surge

- sway

- yaw

Velocities may be expressed in a vessel fixed coordinate system $B$, i.e.

$$
v=\left(\begin{array}{l}
u \\
v \\
r
\end{array}\right) .
$$

These velocities cannot be integrated in $B$ to give position and orientation, but will have to be transformed to an earth fixed (inertial) frame of reference, $E$, i.e.

$$
\begin{aligned}
\dot{\boldsymbol{\eta}} & =\left(\begin{array}{l}
\dot{x} \\
\dot{y} \\
\dot{\psi}
\end{array}\right)=\boldsymbol{J}(\psi) \boldsymbol{v} \\
& =\left(\begin{array}{ccc}
\cos \psi & -\sin \psi & 0 \\
\sin \psi & \cos \psi & 0 \\
0 & 0 & 1
\end{array}\right)\left(\begin{array}{l}
u \\
v \\
r
\end{array}\right) .
\end{aligned}
$$

\subsection{Low frequency vessel model}

A model of the form

$$
\boldsymbol{M} \dot{v}_{L}+\boldsymbol{D} v_{L}=\tau_{L}+\boldsymbol{w}_{L}
$$

is assumed, where

$$
v_{L}=\left(\begin{array}{l}
u_{L} \\
v_{L} \\
r_{L}
\end{array}\right)
$$


is the low frequency velocity vector, $\tau_{L}$ is a vector of control forces and moment, and $\omega_{L}$ is a vector (of zero-mean Gaussian white noise processes) describing unmodeled dynamics and disturbances.

Suppose $\boldsymbol{\eta}_{d}=\left(x_{d}, y_{d}, \psi_{d}\right)^{\mathrm{T}}$ is the desired position and orientation, $\psi \approx \psi_{d}$, and the earth fixed frame of reference is oriented so that $\psi_{d}=0$. Then $\boldsymbol{J}(\psi) \approx \boldsymbol{I}$.

This gives the following state space model:

where

$$
\dot{\boldsymbol{x}}_{L}=\boldsymbol{A}_{L} \boldsymbol{x}_{L}+\boldsymbol{B}_{L} \tau_{L}+\boldsymbol{E}_{L} \boldsymbol{w}_{L}
$$

$$
\begin{aligned}
& \boldsymbol{x}_{L}=\left(x_{L}, y_{L}, \psi_{L}, u_{L}, v_{L}, r_{L}\right)^{\mathrm{T}}, \\
& \boldsymbol{A}_{L}=\left(\begin{array}{cc}
\mathbf{0} & \boldsymbol{I} \\
\mathbf{0} & -\boldsymbol{M}^{-1} \boldsymbol{D}
\end{array}\right), \quad \boldsymbol{B}_{L}=\left(\begin{array}{c}
\mathbf{0} \\
\boldsymbol{M}^{-1}
\end{array}\right), \\
& \boldsymbol{E}_{L}=\left(\begin{array}{c}
\mathbf{0} \\
\boldsymbol{M}^{-1}
\end{array}\right) .
\end{aligned}
$$

\subsection{Wave model}

A model of the environment which induces oscillatory movement of the vessel is now included. This model is used both in the design of an LQG based controller and in the performance evaluation.

A linear approximation to the Pierson-Moskowitz spectrum (Faltinsen 1990, Fossen 1994) is given by

$$
h(s)=\frac{y_{h}}{u_{h}}(s)=\frac{2 K_{w} \zeta \omega_{0} s}{s^{2}+2 \zeta \omega_{0} s+\omega_{0}^{2}} .
$$

In this model $K_{\omega}$ is the intensity, $\omega_{0}$ is the wave frequency and $\zeta$ is a damping factor. This gives the following model for the wave disturbances in the three degrees of freedom:

$$
\begin{aligned}
& \dot{\xi}_{x}=x_{H} \\
& \dot{x}_{H}=-2 \zeta \omega_{0} x_{H}-\omega_{0}^{2} \xi_{x}+2 K_{w x} \zeta \omega_{0} w_{x} \\
& \dot{\xi}_{y}=y_{H} \\
& \dot{y}_{H}=-2 \zeta \omega_{0} y_{H}-\omega_{0}^{2} \xi_{y}+2 K_{w y} \zeta \omega_{0} w_{y} \\
& \dot{\xi}_{\psi}=\psi_{H} \\
& \dot{\psi}_{H}=-2 \zeta \omega_{0} \psi_{H}-\omega_{0}^{2} \xi_{\psi}+2 K_{w \psi} \zeta \omega_{0} w_{\psi}
\end{aligned}
$$

this can be written as

where

$$
\dot{\boldsymbol{x}}_{H}=\boldsymbol{A}_{H} \boldsymbol{x}_{H}+\boldsymbol{E}_{H} \boldsymbol{w}_{H},
$$

$$
\begin{aligned}
\boldsymbol{x}_{H} & =\left(\xi_{x}, \xi_{y}, \xi_{\psi}, x_{H}, y_{H}, \psi_{H}\right)^{\mathbf{T}}, \\
\boldsymbol{w}_{H} & =\left(w_{x}, w_{y}, w_{\psi}\right)^{\mathbf{T}} .
\end{aligned}
$$

It is assumed that $\boldsymbol{w}_{H}$ is a vector of zero mean Gaussian white noise processes and

$$
E\left\{\boldsymbol{w}_{H}(t) \boldsymbol{w}_{H}^{\mathrm{T}}(\tau)\right\}=\boldsymbol{I}_{3 \times 3} \delta(t-\tau) .
$$



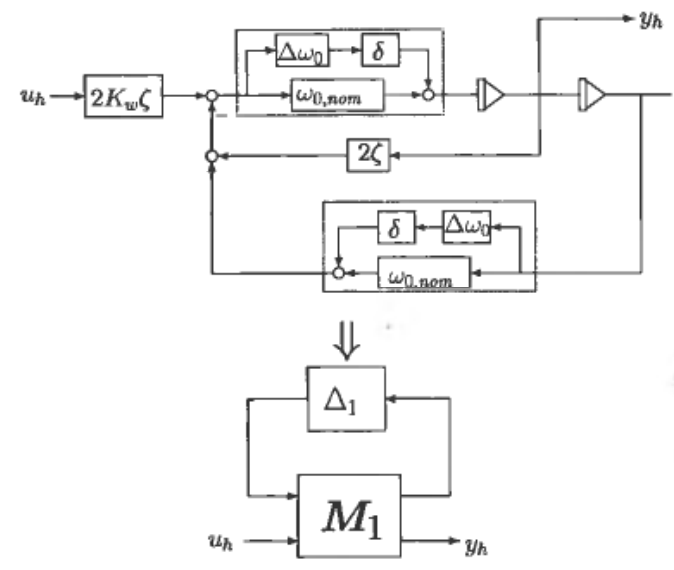

Figure 1. Uncertainty description in the wave disturbance model.

\subsection{Measurements}

The measured position and orientation is assumed to be a linear combination of the low frequency vessel model and the wave induced motion, i.e.

$$
y=C_{L} x_{L}+C_{H} x_{H}+v,
$$

where

$$
C_{L}=\left(\begin{array}{ll}
I_{3 \times 3} & \mathbf{0}_{3 \times 3}
\end{array}\right), \quad C_{H}=\left(\begin{array}{ll}
0_{3 \times 3} & I_{3 \times 3}
\end{array}\right),
$$

and $v$ is zero mean Gaussian white noise.

\subsection{Uncertainty description in the wave disturbance model}

It is assumed that the dominating wave frequency $\omega_{0}$ varies within a band, i.e.

$$
\omega_{0} \in\left(\omega_{0, \text { nom }}-\Delta \omega_{0}, \omega_{0, \text { nom }}+\Delta \omega_{0}\right) \text {. }
$$

As can be seen from Fig. 1, this can be modeled with a real uncertainty block

$$
\Delta_{1}=\left(\begin{array}{ll}
\delta & 0 \\
0 & \delta
\end{array}\right)
$$

Generalizing this, it is seen that the uncertainty in three degrees of freedom can be represented with

such that

$$
\Delta_{1}=\Delta_{2}=\Delta_{3}=\delta \boldsymbol{I}_{2 \times 2},
$$

$$
\Delta=\delta \boldsymbol{I}_{6 \times 6},
$$

and $\delta \in(-1,1)$. This implies that equal frequency variations in the surge, sway and yaw degrees of freedom are assumed.

\section{Robust performance}

\subsection{Robust performance and performance weights}

Consider the block diagram in Fig. 2. Variables with a tilde, $(\approx)$, are scaled according to Appendix B. Define

$$
\begin{aligned}
& \boldsymbol{z}_{\boldsymbol{e}}=\boldsymbol{T}_{z_{e} w_{H}} \boldsymbol{w}_{H}+\boldsymbol{T}_{z_{e} \eta_{d}} \tilde{\boldsymbol{\eta}}_{d}, \\
& \boldsymbol{z}_{\tau}=\boldsymbol{T}_{z_{\tau} w_{H}} \boldsymbol{w}_{H}+\boldsymbol{T}_{z_{\tau} \eta_{d}} \tilde{\boldsymbol{\eta}}_{d} .
\end{aligned}
$$




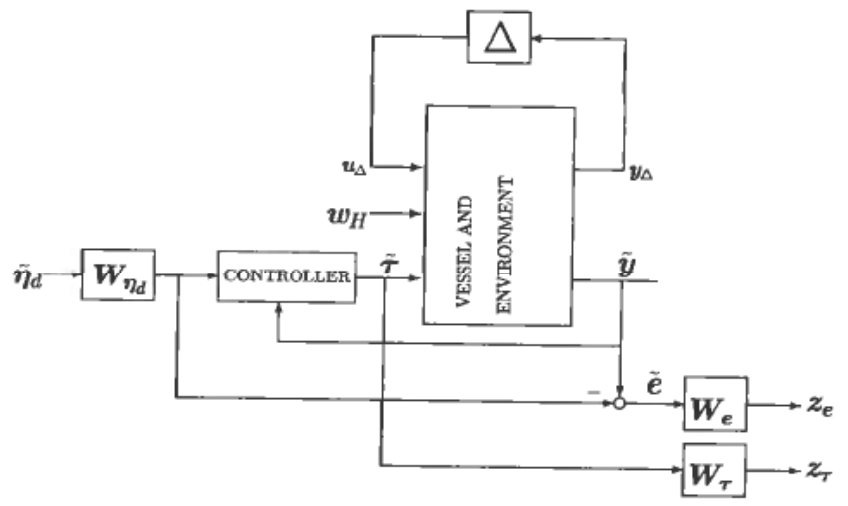

Figure 2. Control system with weights on reference signal and performance. There is uncertainty in the environment model.

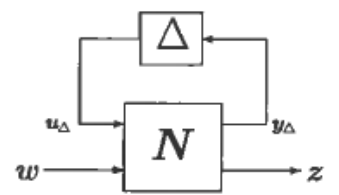

Figure 3. Structure for assessing robust performance.

Let

$$
\boldsymbol{w}=\left(\begin{array}{c}
\boldsymbol{w}_{H} \\
\boldsymbol{\eta}_{d}
\end{array}\right), \quad z=\left(\begin{array}{l}
\boldsymbol{z}_{e} \\
\boldsymbol{z}_{\tau}
\end{array}\right),
$$

such that Fig. 2 can be transformed into Fig. 3. With a proper scaling of inputs and outputs, robust performance is defined as follows:

\section{Definition 1 (Robust performance)}

The system in Fig. 3 is said to exhibit robust performance if i.e.

$$
\|z(j \omega)\|<1, \quad \forall \omega, \boldsymbol{w}, \Delta \mid\|\boldsymbol{w}(j \omega)\| \leqslant 1, \quad \bar{\sigma}\{\Delta(j \omega)\} \leqslant 1,
$$

$$
\|T\|_{\infty} \leqslant 1
$$

when $z=T w$.

Remark: From equations (14)-(15) it is recognized that

$$
\boldsymbol{T}=\left(\begin{array}{ll}
\boldsymbol{T}_{z_{e} w_{H}} & \boldsymbol{T}_{z_{e} \eta_{d}} \\
\boldsymbol{T}_{z_{\tau} w_{H}} & \boldsymbol{T}_{z_{\tau} \eta_{d}}
\end{array}\right) .
$$

Now weights on control error and control input as shown in Fig. 2 will be considered. Let

and

$$
\boldsymbol{W}_{e}(s)=10^{2} \frac{1+10^{2} s}{\left(1+10^{4} s\right)(1+10 s)} \boldsymbol{I}_{3 \times 3}=w_{e}(s) \boldsymbol{I}_{3 \times 3}
$$

$$
\boldsymbol{W}_{\tau}(s)=\rho \frac{s}{1+10 s} \boldsymbol{I}_{3 \times 3}=w_{\tau}(s) \boldsymbol{I}_{3 \times 3},
$$




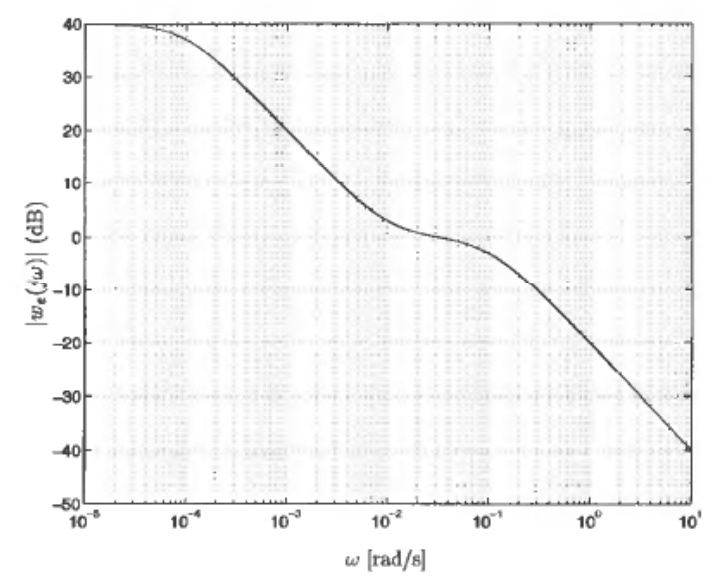

Figure 4. Weight on control error.

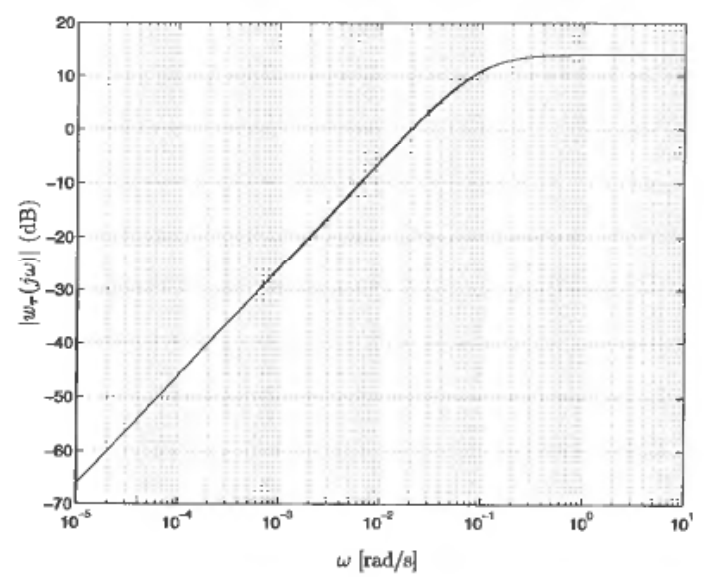

Figure 5. Weight on control input.

where

$$
\rho=50 .
$$

These weights indicate the following:

- small stationary control error allowed

- no weight on high frequency control error

- no weight on stationary control input

- weight on high frequency control input to penalize thruster modulation

A weight on the reference signal is included. This indicates that the reference signal has low frequency components only. Let

$$
\boldsymbol{W}_{\eta d}(s)=\frac{1}{1+10^{4} s} \boldsymbol{I}_{3 \times 3} .
$$

Plots of $\left|w_{e}(j \omega)\right|$ and $\left|w_{\tau}(j \omega)\right|$ are shown in Figs. 4 and 5. 


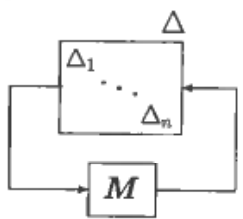

Figure 6. Matrices in definition of the structured singular value.

\subsection{Analysis of robust performance using the structured singular value}

The structured singular value will now be studied. The SSV will be used due to the block diagonal uncertainty description.

\section{Definition 2 (Structured singular value, $\mu$, (Doyle 1982))}

Let $\boldsymbol{X}_{\delta}$ denote the set of stable, block diagonal perturbations (see Fig. 6) with a particular structure, where the diagonal elements satisfy $\left\|\Delta_{j}\right\|_{\infty} \leqslant \delta$ then,

$$
\mu_{\Delta}(\boldsymbol{M})=\left\{\begin{array}{l}
0 \text { if no } \Delta \in \boldsymbol{X}_{\infty} \text { solves } \operatorname{det}(\boldsymbol{I}-\boldsymbol{M} \Delta)=0, \\
\left(\min _{\Delta \in \boldsymbol{X}_{\infty}}\{\bar{\sigma}(\Delta) \mid \operatorname{det}(\boldsymbol{I}-\boldsymbol{M} \Delta)=0\}\right)^{-1} \text { otherwise. }
\end{array}\right.
$$

Computer software exists to compute the structured singular value (Balas et al. 1991). In particular, new versions of the software can compute $\mu$ with a mixed $\mathrm{real} / \mathrm{complex}$ uncertainty structure.

A recent tutorial on the structured singular value can be found in Packard and Doyle (1993). Details on $\mu$ analysis with real parametric uncertainty can be found in Young et al. (1991).

Now, consider the following sentences which will prove useful.

\section{Lemma I (Robust stabilitv)}

The system in Fig. 6 is stable for all $\Delta \in \boldsymbol{X}_{1}$ if and only if

$$
\mu_{\Delta}\{\boldsymbol{M}(j \omega)\}<1, \quad \forall \omega .
$$

Proof: See Maciejowski (1989).

\section{Lemma 2 (Robust performance)}

The system in Fig. 3 has robust performance if and only if

$$
\mu_{\Delta}\{N(j \omega)\}<1, \quad \forall \omega,
$$

where

$$
\hat{\Delta}=\left(\begin{array}{cc}
\Delta & \mathbf{0} \\
\mathbf{0} & \Delta_{p}
\end{array}\right)
$$

Proof: See Maciejowski (1989).

Note that robust performance is ensured by adding a fictitious uncertainty block $\Delta_{p}$.

\section{Evaluation of performance robustness for different controller structures}

Two controller structures are now investigated, a PD solution and a solution using state estimation and optimal control. Robust performance is analysed using methods. uncertainty description, and performance weights from the previous sections. 


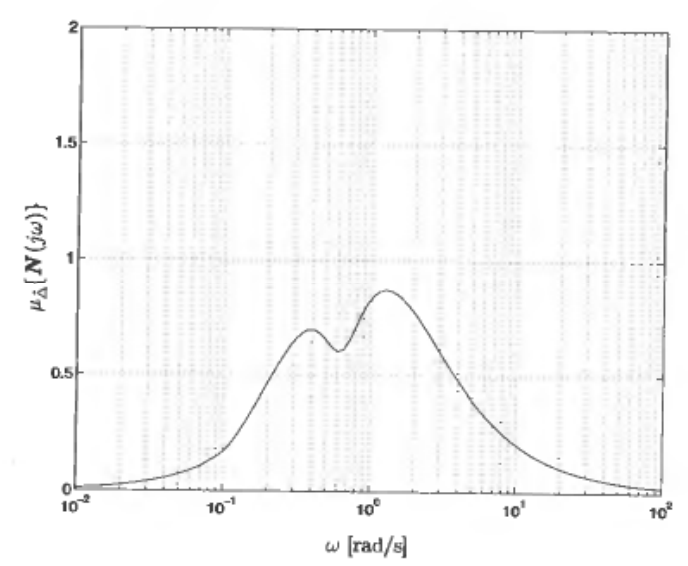

Figure 7. $\mu_{\hat{\Delta}}\{N(j \omega)\}$ with $\Delta \omega_{0}=0$ and PD controller.

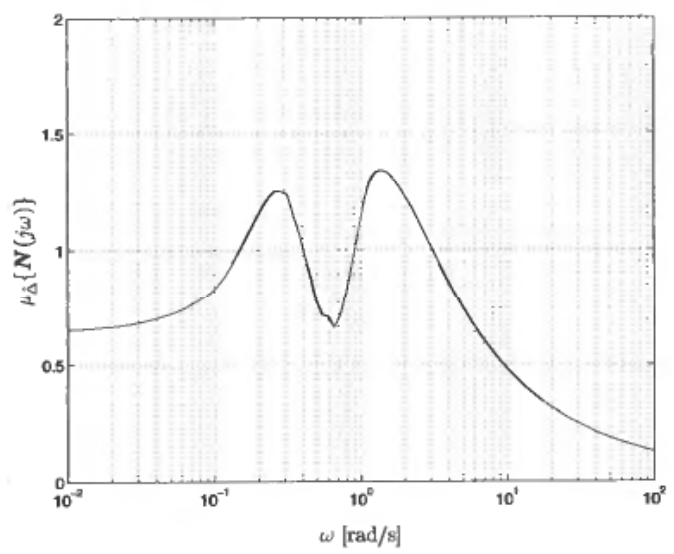

Figure 8. $\mu_{\hat{\Delta}}\{N(j \omega)\}$ with $\Delta \omega_{0}=0.4 \mathrm{rad} / \mathrm{s}$ and PD controller.

Both controllers are tuned to give a closed loop bandwidth of approx. $0 \cdot 1 \mathrm{rad} / \mathrm{s}$. Nominal dominating wave frequency is assumed to be $\omega_{0}=2 \pi / 10 \approx 0.6 \mathrm{rad} / \mathrm{s}$.

\subsection{PD solution}

A PD controller combined with a notch filter (for attenuation of wave disturbances) will be used in each degree of freedom.

The control input (scaled) is given by

$$
\tilde{\boldsymbol{\tau}}=\boldsymbol{C}_{P D}\left[\tilde{\boldsymbol{\eta}}_{d}-\tilde{\boldsymbol{y}}\right],
$$

where

$$
C_{P D}(s)=\operatorname{diag}\left\{c_{x}(s) \quad c_{y}(s) \quad c_{\psi}(s)\right\},
$$

with

$$
c_{j}(s)=K_{p, j} \frac{1+T_{d, j} s}{1+x_{j} T_{d, j} s} \frac{1+2 \zeta_{n} \frac{s}{\omega_{0}}+\left(\frac{s}{\omega_{0}}\right)^{2}}{1+2 \zeta_{n} \frac{s}{\omega_{0}}+\left(\frac{s}{\omega_{0}}\right)^{2}} .
$$

Numerical values are given in Table 1 . 
Now $\Delta=\delta \boldsymbol{I}_{6 \times 6}$ and $\Delta_{p} \in \mathbb{C}^{6 \times 6}$. Figs. 7 and 8 show $\mu_{\Delta}\{N(j \omega)\}$ with $\Delta \omega_{0}=0$ and $\Delta \omega_{0}=0.4 \mathrm{rad} / \mathrm{s}$, respectively.

\subsection{State estimation and optimal control}

States

$$
\hat{\tilde{\boldsymbol{x}}}=\left(\begin{array}{c}
\hat{\tilde{\boldsymbol{x}}}_{L} \\
\hat{\tilde{\boldsymbol{x}}}_{H}
\end{array}\right)
$$

are estimated using a Kalman filter. Tuning parameters are

and

$$
\begin{aligned}
E\left\{\boldsymbol{w}_{H}(t) \boldsymbol{w}_{H}^{\mathrm{T}}(\tau)\right\} & =\boldsymbol{I}_{3 \times 3} \delta(t-\tau), \\
E\left\{\tilde{\boldsymbol{w}}_{L}(t) \tilde{\boldsymbol{w}}_{L}^{\mathrm{T}}(\tau)\right\} & =\boldsymbol{W}_{L} \delta(t-\tau),
\end{aligned}
$$

Feedback is taken from the estimated low frequency state, i.e.

$$
\tilde{\boldsymbol{\tau}}=\boldsymbol{K}_{\text {contr }}\left[\left(\begin{array}{c}
\tilde{\boldsymbol{\eta}}_{d} \\
\mathbf{0}_{3 \times 1}
\end{array}\right)-\hat{\tilde{\boldsymbol{x}}}_{L}\right]
$$

$\boldsymbol{K}_{\text {contr }}$ is computed using the performance index

$$
J=\int_{0}^{\mathrm{T}}\left(\hat{\tilde{\boldsymbol{x}}}_{L}^{\mathrm{T}} Q \hat{\overline{\boldsymbol{x}}}_{L}+\tilde{\boldsymbol{\tau}}^{\mathrm{T}} \boldsymbol{P} \tilde{\tau}\right) \mathrm{d} t .
$$

Numerical values are given in Table 2.

Figures 9 and 10 show $\mu_{\Delta}\{N(j \omega)\}$ with $\Delta \omega_{0}=0$ and $\Delta \omega_{0}=0.4 \mathrm{rad} / \mathrm{s}$, respectively.

\section{Discussion and conclusions}

First note that the control design examples did not include integral action. In practice this would be used to give the required high gain at low frequencies. In addition, the PD control example should include filtering to avoid feedback of high frequency measurement noise. However, this would not change the robustness analysis significantly.

Figures 7 and 9 may be interpreted as plots of nominal performance, which is satisfied-with the proposed performance weights. When uncertainty is imposed, Figs. 8 and 10 show that robust performance is not achieved. The structured singular value $\mu_{\Delta}\{N(j \omega)\}$ crosses 1 both at frequencies above and below the nominal dominating wave frequency. This indicates that robust performance is not obtained with the selected controller parameters, and that thruster modulation may be experienced. It also suggests that tracking the dominating wave frequency is indeed reasonable.

In the analysis, it has been assumed that only the frequency of the dominating wave induced vessel motion is changed, and that the intensity (amplitude) of that motion remains constant. For this to be true both the dominating wave frequency and wave intensity would have to change. This is due to the fact that wave excitations are filtered through the vessel dynamics to give the vessel motion.

Other uncertainty factors could easily have been included in the analysis, such as uncertainty in the intensity of the wave induced motion or uncertainty in the thrust allocation algorithm.

It is common to include blocks indicating uncertainty due to high frequency 


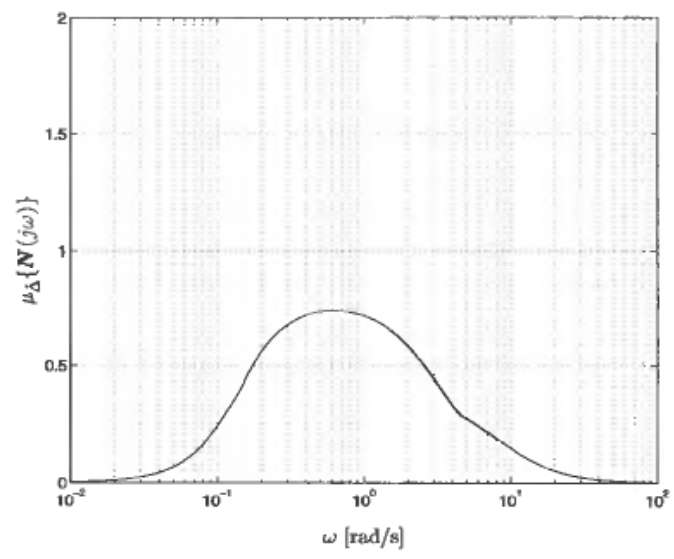

Figure 9. $\mu_{\Delta}\left\{N\left(j \omega_{0}\right)=0\right.$ and state estimator/optimal control.

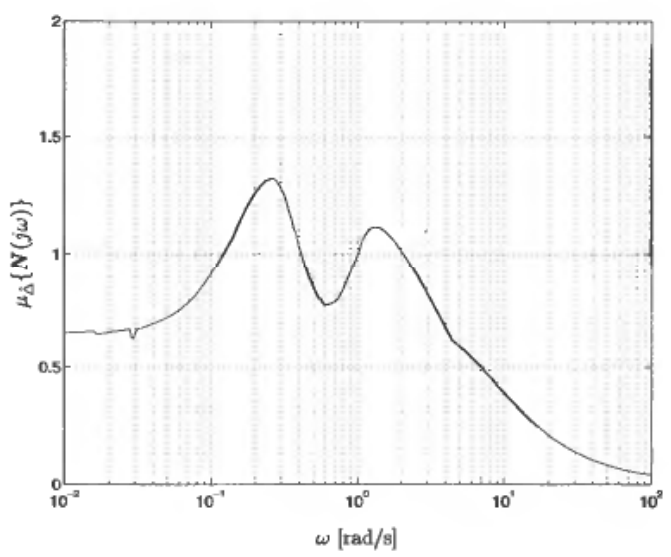

Figure 10. $\mu_{\partial}\{N(j \omega)\}$ with $\Delta \omega_{0}=0.4 \mathrm{rad} / \mathrm{s}$ and state estimator/optimal control.

unmodeled dynamics. Using the structured singular value, such general model uncertainty can be combined with parametric uncertainty (as in the present analysis).

One important point to notice, is that robustness requirements in general are independent of a particular designed controller. In the two control designs proposed in Section 4 uncertainty was not considered explicitly.

\section{ACKNOWLEDGMENT}

This work was supported in part by Simrad Albatross AS.

\section{Appendix A. Model parameters}

Data for a supply vessel is given in Fossen (1994)

$$
\begin{aligned}
M^{\prime \prime} \dot{v}^{\prime \prime}+D^{\prime \prime} v^{\prime \prime} & =\tau^{\prime \prime}, \\
\dot{v}^{\prime \prime} & =A^{\prime \prime} v^{\prime \prime}+B^{\prime \prime} v^{\prime \prime},
\end{aligned}
$$

where all quantities with double prime $(\cdot)^{\prime \prime}$ are scaled with the so-called bis system.

These non-dimensional quantities are scaled to physical values using three parameters: 


$$
\begin{array}{cl}
L=76.2 \mathrm{~m} & \text { length } \\
m=4 \cdot 10^{6} \mathrm{~kg} & \text { mass } \\
g=9.81 \mathrm{~m} / \mathrm{s}^{2} & \text { acceleration of gravity }
\end{array}
$$

The following values are estimated:

$$
\begin{gathered}
M^{\prime \prime}=\left(\begin{array}{rrr}
1.1274 & 0 & 0 \\
0 & 1.8902 & -0.0744 \\
0 & -0.0744 & 0.1278
\end{array}\right), \\
A^{\prime \prime}=\left(M^{\prime \prime}\right)^{-1}\left(\begin{array}{rrr}
-0.0318 & 0 & 0 \\
0 & -0.0602 & -0.0618 \\
0 & -0.0075 & -0.2454
\end{array}\right) M^{\prime \prime}
\end{gathered}
$$

\section{Appendix B. Scaling of inputs and outputs}

It would have been possible to work with a dimensionless model in the bis system. However, it seems reasonable to use characteristic parameters in the problem at hand. The following characteristic deviations in inputs, outputs, disturbances etc. are selected as scaling parameters:

$$
\begin{array}{rlrl}
\left|\Delta \boldsymbol{\tau}_{x}\right|_{\max } & =7.3 \cdot 10^{5} \mathrm{~N} & & \\
\left|\Delta \tau_{y}\right|_{\max } & =3.7 \cdot 10^{5} \mathrm{~N} & & \\
\left|\Delta \tau_{\psi}\right|_{\max } & =1.1 \cdot 10^{7} \mathrm{Nm} & & \\
\left|\Delta \boldsymbol{w}_{H}\right|_{\max } & =1.0 & & \\
\left|\Delta \boldsymbol{e}_{x}\right|_{\max } & =5 \mathrm{~m} & \left|\Delta \dot{\boldsymbol{e}}_{x}\right|_{\max }=0.5 \mathrm{~m} / \mathrm{s} \\
\left|\Delta \boldsymbol{e}_{y}\right|_{\max } & =5 \mathrm{~m} & & \left|\Delta \dot{\boldsymbol{e}}_{y}\right|_{\max }=0.5 \mathrm{~m} / \mathrm{s} \\
\left|\Delta \boldsymbol{e}_{\psi}\right|_{\max } & =3^{\circ} & & \left|\Delta \dot{\boldsymbol{e}}_{\psi}\right|_{\max }=0.3 \% \mathrm{~s}
\end{array}
$$

With these scaling parameters, a control input of 1 will indicate saturation (maximum achievable thrust). A value of 1 in measurement of $x$ position then corresponds to a physical measurement of 5 meters.

Maximum forces in the surge and sway directions and maximum torque about the yaw axis are estimated from parameters in Fossen (1994).

\section{Appendix C. Controller parameters}

\section{C.1. PD controller}

\begin{tabular}{cccc}
\hline$j$ & $K_{p, j}$ & $T_{d, j}$ & $\alpha_{j}$ \\
\hline$x$ & $1 / 20$ & $33 \cdot 33$ & $0 \cdot 1$ \\
$y$ & $1 / 6$ & $33 \cdot 33$ & $0 \cdot 1$ \\
$\psi$ & $1 / 35$ & $33 \cdot 33$ & $0 \cdot 1$ \\
\hline
\end{tabular}

Table 1. Numerical values in PD controller.

$\zeta_{n}=0 \cdot 3, \zeta_{d}=1 \cdot 5, \omega_{0}=2 \pi / 10$. the controller is designed with respect to the scaled plant, i.e. the controller gains are dimensionless. 


\section{C.2. State estimation and optimal control}

Let

$$
\boldsymbol{K}_{w}=\left(\begin{array}{lll}
\tilde{\boldsymbol{K}}_{w x} & \tilde{\boldsymbol{K}}_{w y} & \tilde{\boldsymbol{K}}_{w \psi}
\end{array}\right) .
$$

This tuning parameter is a function of sea state. Here a reasonable example value is selected. $K_{w x}, K_{w y}$ and $K_{w \psi}$ are scaled using the values for characteristic deviation in the surge, sway and yaw directions.

$$
\begin{aligned}
& \boldsymbol{Q}=10^{2} \boldsymbol{I}_{6 \times 6} \\
& \boldsymbol{P}=\boldsymbol{I}_{3 \times 3} \\
& \boldsymbol{W}_{\mathrm{L}}=\mathbf{5} \cdot 10^{-3} \boldsymbol{I}_{3 \times 3} \\
& \boldsymbol{V}=10^{-2} \boldsymbol{I}_{3 \times 3} \\
& \boldsymbol{K}_{\omega}=\left(\begin{array}{lll}
0.6 & 0.4 & 1.33
\end{array}\right)
\end{aligned}
$$

Table 2. Numerical values in state estimation/optimal control.

Note that $w_{\mathrm{L}}$ is scaled using values for maximum thrust.

\section{REFERENCES}

Balas, G. J., Doyle, J. C., Glover, K., PaCKard, A. and Smith, R. (1991). $\mu$-Analysis and Synthesis Toolbox. User's Guide (The MathWorks and MUSYN).

Balchen, J. G., Jenssen, N. A. and Saelid, S. (1976). Dynamic positioning using Kalman filtering and optimal control theory. In: Automation In Offshore Oil Field Operation. (North-Holland Publishing Company), pp. 183-187.

Balchen, J. G., Jenssen, N. A., Mathisen, E. and Saelid, S. (1980). A dynamic positioning system based on Kalman filtering and optimal control. Modeling, Identification and Control, 1, 135-163.

DoyLE, J. C. (1982). Analysis of feedback systems with structured uncertainties. IEE Proceedings, 129, 242-250.

Doyle, J. C. and Stein, G. (1981). Multivariable feedback design: concepts for a classical/ modern synthesis. IEEE Transactions on Automatic Control, AC-26, 4-16.

Faltinsen, O. M. (1990). Sea Loads on Ships and Offshore Structures (Cambridge University Press, Cambridge).

FAY, H. (1990) Dynamic positioning systems: principles, design and applications (Technip., Paris).

Fossen, T. I. (1994). Guidance and Control of Ocean Vehicles (John Wiley \& Sons, Chichester). Grimble, M. J. and PATtON, R. J. (1980). The design of dynamic ship positioning control systems using stochastic optimal control theory. Optimal Control Applications \& Methods, 1, 167-202.

Grimble, M. J., PAtton, R. J. and Wise, D. A. (1980). Use of Kalman filtering techniques in dynamic ship-positioning systems. IEE Proceedings, 127(3), 93-102.

MACIEJOWSKı, J. M. (1989). Multivariable Feedback Design (Addison-Wesley, Wokingham, UK).

Morgan, M. J. (1978). Dynamic positioning of offshore vessels (The Petroleum Publishing Company, Tulsa, Oklahoma).

PACKARD, A. and DoYle, J. (1993). The complex structured singular value. Automatica, 29, 71-109.

SFlid, S., JensSEN, N. A. and BAlChen, J. G. (1983) Design and analysis of a dynamic positioning system based on Kalman filtering and optimal control. IEEE Transactions on Automatic Control, AC-28(3), 331-339.

SKOGESTAD, S. (1994) Multivariable Feedback Control-Analysis and design using frequencydomain methods. Department of Chemical Engineering, University of Trondheim, Trondheim, Norway. Lecture notes. To be published by John Wiley \& Sons.

Young, P. M., Newlin, M. P., and Doyle, J. C. (1991) $\mu$ Analysis with real parametric uncertainty. In: Proceedings of the 30th Conference on Decision and Control (IEEE. Brighton, England) pp. 1251-1256. 